\title{
Fuzzy Rules to Improve Traffic Light Decisions in Urban Roads
}

\author{
J. A. Castán Rocha ${ }^{1}$, S. Ibarra Martínez ${ }^{1}$, J. Laria Menchaca ${ }^{1}$, J. D. Terán Villanueva ${ }^{1}$, \\ M. G. Treviño Berrones' ${ }^{1}$, J. Pérez Cobos ${ }^{1}$, D. Uribe Agundis ${ }^{2}$
}

${ }^{1}$ Faculty of Engineering, Autonomous University of Tamaulipas, Ciudad Victoria, Mexico

${ }^{2} \mathrm{TecNM} /$ Instituto Tecnológico de La Laguna, Torreon, Mexico

Email: sibarram@uat.edu.mx

How to cite this paper: Castán Rocha, J.A., Ibarra Martínez, S., Laria Menchaca, J., Terán Villanueva, J.D., Treviño Berrones, M.G., Pérez Cobos, J. and Uribe Agundis, D. (2018) Fuzzy Rules to Improve Traffic Light Decisions in Urban Roads. Journal of Intelligent Learning Systems and Applications, 10, 36-45.

https://doi.org/10.4236/jilsa.2018.102003

Received: January 10, 2018

Accepted: May 19, 2018

Published: May 22, 2018

Copyright () 2018 by authors and Scientific Research Publishing Inc. This work is licensed under the Creative Commons Attribution International License (CC BY 4.0).

http://creativecommons.org/licenses/by/4.0/

\begin{abstract}
Many researchers around the world are looking for developing techniques or technologies that cover traditional and recent constraints in urban traffic control. Normally, such traffic devices are facing with a large scale of input data when they must to response in a reliable, suitable and fast way. Because of such statement, the paper is devoted to introduce a proposal for enhancing the traffic light decisions. The principal goal is that a semaphore can provide a correct and fluent vehicular mobility. However, the traditional semaphore operative ways are outdated. We present in a previous contribution the development of a methodology capable of improving the vehicular mobility by proposing a new green light interval based on road conditions with a CBR approach. However, this proposal should include whether it is needed to modify such light duration. To do this, the paper proposes the adaptation of a fuzzy inference system helping to decide when the semaphore should try to fix the green light interval according to specific road requirements. Some experiments are conducted in a simulated environment to evaluate the pertinence of implementing a decision-making before the CBR methodology. For example, using a fuzzy inference approach the decisions of the system improve almost $18 \%$ in a set of 10,000 experiments. Finally, some conclusions are drawn to emphasize the benefits of including this technique in a methodology to implement intelligent semaphores.
\end{abstract}

\section{Keywords}

Fuzzy Inference System, Urban Traffic Control, Vehicular Mobility, Intelligent Transport System

\section{Introduction}

One of the most pursuit aspects to be improved in modern cities is its transit in- 
frastructures. A traffic infrastructure is a dynamic and open scenario shaped by different kind of information which is used to make trustworthy and reliable decisions. In Mexico, the traditional way of controlling a junction is by using a traffic light signal. Such control was functional and suitable for the past. The number of cars and other relevant factors that affect the uses of the road are representing a technological challenge for researchers. In previous work [1] presented a methodology that makes an evaluation of the green light time based on the volume of cars to modify the duration of such light in the next phase of the semaphore. However, it was observed that sometimes the change in the green light duration was made and this change was not really significant to increase the level of service of the road. As a first conclusion, the fact of incurring in a non-adequate recommendation could cause both noise in the previously synchronized phase of the junction and increase the computational cost associated to the procedure of the system. The relevance of improving the way of adjusting the traffic light signals is quite needed due to current road constraints. For that reason, the paper proposes to include a prior review of some road aspects in order to decide if a modification of the green light interval could improve the service level of the junction. A fuzzy inference system is evaluated in order to corroborate the above statement.

A Fuzzy inference system can define whether semaphore must modify the time assigned to its green light with a high level of reliability. In fact, this technique is widely implemented to provide decisions under uncertain information. For instance, the fuzzy logic is a completely probabilistic technique defined by [2] as an extension of the traditional logic that uses concepts of ownership of an item which allows to measure the degree of membership of this element within a particular class and that has as main advantage the using linguistic terms to express knowledge, just as the human do in a natural way.

In [3], the author grounds its arguments about safety in construction industries on a proper and opportune occupational risk assessment. Recent research concludes that such evaluation is quite difficult due to inadequate or uncertain data. For thus, the authors propose a Takani-Sugeno fuzzy inference systems to cover information lacks. The risk factor and controlling factors for accidental injuries are considered as input parameters. Analytical hierarchy process and subtractive clustering techniques are used both to evaluate safety levels of each type of injury and to reduce the number of rules in the ANFIS, respectively. To the end, a hybrid learning process is implemented to prove all the parameters corresponding to the input variables. The methodology has been applied to few selected construction sites in India.

Moreover, in [4] for the detection of fire use a diffuse model able to discern the areas that present a fire situation by recognition of colors. In this approach, the system generates a fuzzy search to locate fire, which handles processes only to the classification of binary map of the pixels of the background of the image. The fuzzy inference system follows a Mamdani process and consists of four 
stages: defuzzification of the variable, evaluation rules, composition and defuzzification, all based on the trapezoidal membership functions. The results show an improvement of almost $87 \%$ in comparison with the Bo-Ho Cho and Celik algorithms.

References [5] is proposed a fuzzy rule-based algorithm to evaluate job-satisfaction in an organization. The authors suggest interviews to obtain information about the relationship between effective factors and jobs facets. In particular, the Minnesota Satisfaction Questionnaire is used to obtain values of basis determinants of the respondents. Some fuzzy rules were generated from this information in order to compute the value of job satisfaction using the expert system shell ESPLAN. The proposed approach shows relevant advantages to operate with imperfect information for the evaluation of job satisfaction by using fuzzy logic.

Besides, in [6] a study to show how to put an expert system in practice to organize the security screening process is proposed. Authors highlight the relevance of the expert's opinion and problems involved in the imprecise nature of information to take advantages of fuzzy sets and fuzzy inference system to generate FUPSCA (Fuzzy Passenger Security Control Assessment). It is used to set the parameters of different variables related to the current level of terrorist threat in airports. In conclusion, the paper argues that in a near future, the use of multi-criteria evaluation such as capacity, effectiveness or passenger comfort to manage passenger security control systems will be necessary.

All the aforementioned approaches are evidence of many works oriented to provide effective approaches to solve well-detected lacks in transport area. The main conclusion of these works aims to continue the evaluation of studies on three specific subjects: 1) the interaction among autonomous semaphores; 2) the capability of the system to be robust under uncertain data; and 3) the reliability on the semaphore decisions. About such statement, the paper argues that the implementation of the support decision technique studied appears to be a suitable and robust way to decide when a semaphore must attempt to change the duration of a green light. Different variables can be implicated in this important and dynamic decision. However, FIS method arises as a proper solution to be a filter which avoids that semaphore performs unnecessary actions. Therefore, the motivation of this paper is to strengthen the operatively of a novel methodology for controlling semaphores in an autonomous way using a CBR approach. The remainder of this paper is organized as follows: Section 2 introduces the specific aspects of our proposal. Section 3 presents the results obtained in the experiments. Finally, Section 4 emphasizes the main contributions of the paper and defines some ideas for further progress on this interesting domain.

\section{Fuzzy Inference System in Traffic Control}

Modern cities are looking for offering a suitable traffic service level from different perspectives (i.e., urbanism, town planning or computer science). The idea is 
not only to increase the productiveness of the city but also it is to reach a more comfortable, secure and healthy environment for all the citizens. Among all the particularities of this utopia, roads better administered are one of the most studied topics in the actuality. In this sense, the authors present in [1], a general methodology capable of evaluating some aspects on the road in order to modify the green light interval of a semaphore using a CBR approach. However, one data among the results demonstrate that in occasions modifying such interval is not the appropriate response of the system.

For example, in some cases, the system recommends aggregating 2 or 3 seconds to the green light interval and this action does not reflect an increment in the service level of the analyzed line. In spite of this, it is necessary to evaluate real information related to the road conditions before of initializing the processes of the methodology. To do this, the authors propose to include a FIS technique to define if it is suitable to do a modification in the green light duration of a determined semaphore. This method will allow the system to validate a set of attributes to determine, prior to the start of a green light, if according to the conditions of the road, it will be necessary modify the duration of such light.

To consider a possible change of the green light duration in an isolated 4-lane traffic intersection using a FIS (Fuzzy Inference System), we propose a Mamdani model with five input variables such as:

- Number of incoming cars in each line of this specific road.

- Green light time assigned to the semaphore in its previous intervention in the junction phase.

- Day time in real clock scheduling.

- Type of road that semaphore should administrate.

- Traffic condition at the moment of the FIS decision.

Each of these input variables are represented by a trapezoidal membership function. The response of the model (i.e., determine whether or not to change the duration of the green light) is then characterized by a triangular function as is illustrated in Figure 1. For sake of simplicity, the proposed approach defines a set of attributes for each input variable which are introduced in Table 1. These values were selected and calculated based on previous work presented in [1] using experience of human experts on traffic control.

In this way, the graphical representation of the membership functions of the linguistic attributes for each of the defined variables, by using the MatLab diffuse control is presented in Figure 2.

In order to ensure a proper application of green light times in a semaphore, the output variable called decision is represented by a dichotomous response (YES or NO) (Figure 3). To note, this new system procedure only is in-charge to decide if the semaphore should evaluate whether the green light interval must be modified. Only two answers are defined: "No", which means that according to the FIS, the information provided for the road is not significant enough to indicate that a change in the duration of the green light should reflect a better vehicular 


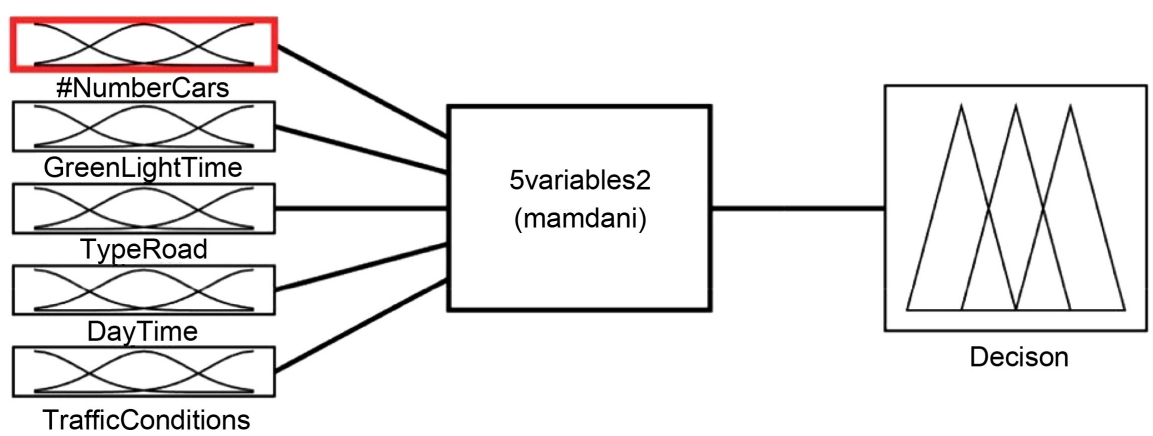

Figure 1. Scheme of the Mamdani model to evaluate semaphore decisions.
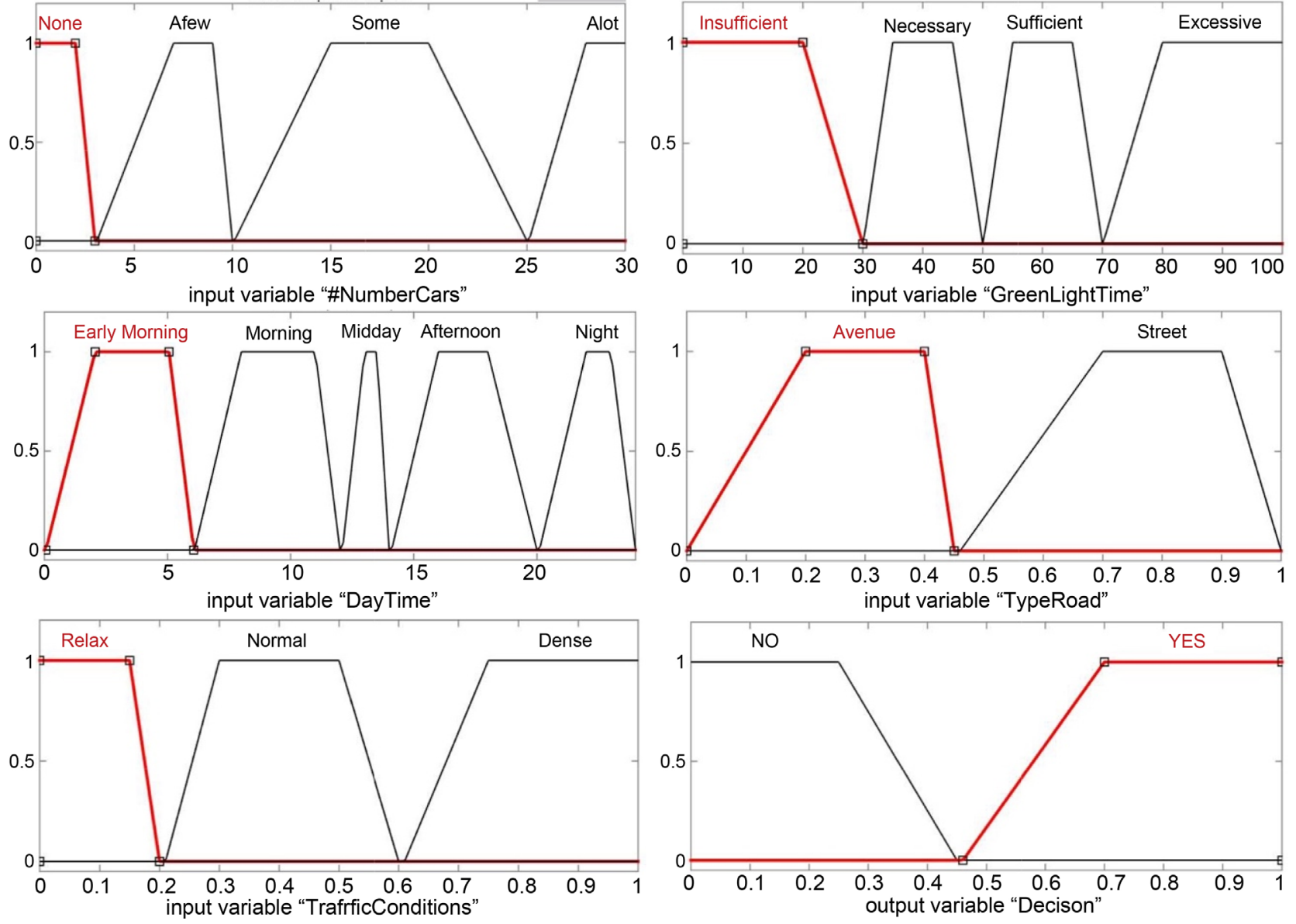

Figure 2. Membership representation of the attributes for each input variable and decision.

mobility. Contrary to this, the "Yes" response represents the membership of the road conditions in which is recommended to do a change in the time of the green light. To express the above, a set of 56 rules was generated, where each variable was combined with the rest, so that all possible combinations were considered, as illustrated in Figure 4 (only 30 rules are presented).

\section{Experimental Results}

All the experiments were developed on a Macbook Pro with the following 


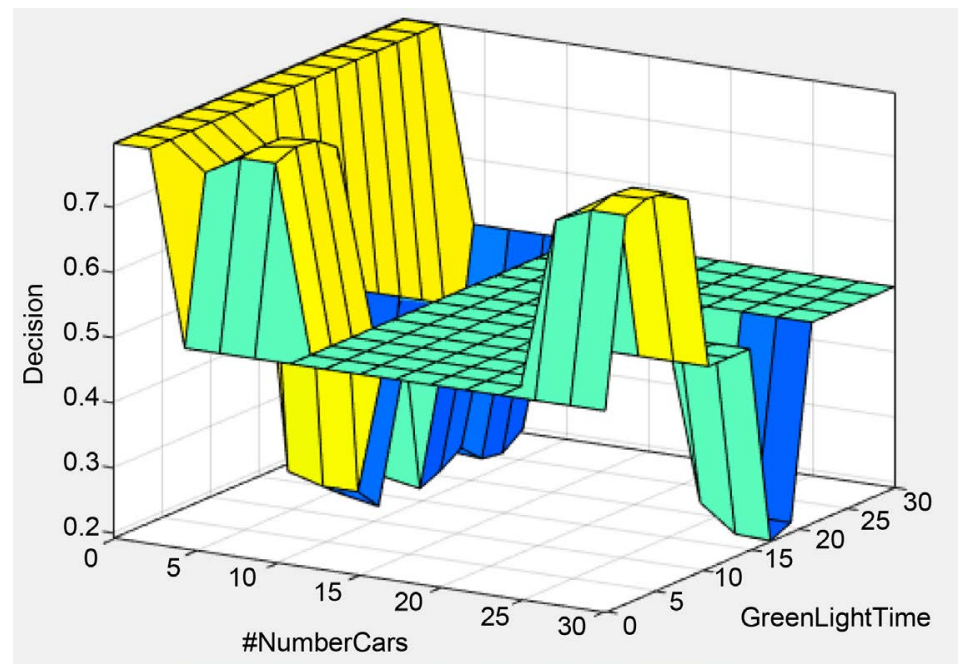

Figure 3. Surface view for number of cars and green light time.

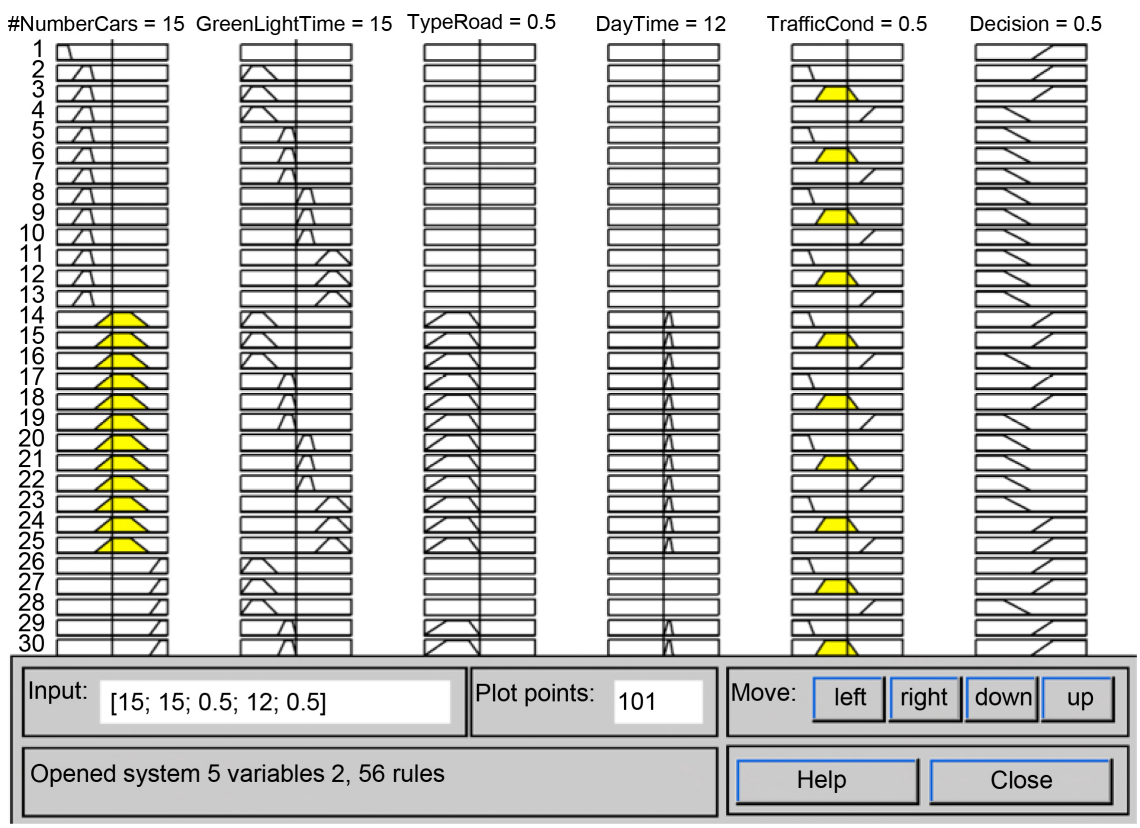

Figure 4. Review of the behavior rules of the diffuse inference system based on the Mamdani model.

specification:

- macOS Sierra OS10.12.6 version,

- Intel Core i7 $2.90 \mathrm{GHz}$ Processor,

- 8 GB $1600 \mathrm{MHz}$ DDR3 memory,

- 1 TB SSD storage.

To validate the efficiency of the generated FIS, a phase of 10,000 control experiments were performed each, taking as reference the representation of an infrastructure of four roads, one of the most used in Mexico (see Figure 5) with the following characteristics:

- Each road has movements in both directions. 


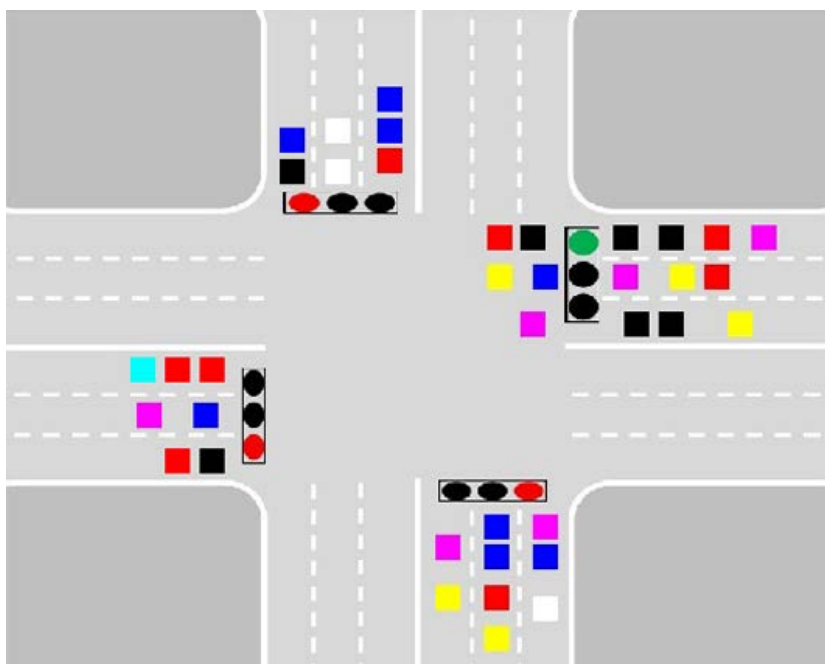

Figure 5. The representation of an infrastructure of four roads used in the test.

Table 1. Relation variable attributes for the fuzzy decision model.

\begin{tabular}{ccc}
\hline VARIABLE & ATTRIBUTE & VALUE \\
\hline \multirow{2}{*}{ Number of Cars } & None & $0-3$ \\
& A Few & $3-10$ \\
& Some & $10-25$ \\
& A lot & 25 or more \\
& Insufficient & $0-30$ \\
Green Light Time & Necessary & $30-50$ \\
& Sufficient & $50-70$ \\
& Excessive & $70-100$ \\
Early Mourning & Mourning & $0-6$ \\
Day Time & Midday & $6-12$ \\
& Afternoon & $12-14$ \\
& Night & $14-20$ \\
Type of Road & Avenue & $20-24$ \\
& Street & $0.00-0.45$ \\
& Relax & $0.45-1.00$ \\
& Normal & $0.00-0.20$ \\
& Dense & $0.21-0.60$ \\
& & $0.61-1.00$ \\
\hline
\end{tabular}

- It can turn left (according to the traffic laws that are regulated in Mexico).

- Continuous right turn (i.e., not necessarily the semaphore should have green light, simply the driver must be cautious).

- Each road has three lanes.

- Average speed of the car $50 \mathrm{~km} / \mathrm{h}$.

- Different types of cars (i.e., sizes and shapes). 
The results are compared against the performance obtained from the system when it modifies the green light time at all turns of the semaphore cycle. In this sense, if the system uses a decision support technique, such as the FIS technique, its performance increases by almost $18 \%$ as shown in Figure 6 .

Another interesting point of view to be noted is the number of experiments each one of the proposed approaches needed to reach stability in the overall system performance. In other words, this data reflects the number of situations that system requires to get sufficient information into their data base in order to reach trustworthy and reliable decisions. For instance, in Figure 7 the computed curve using a sliding window up to the current trial with a smooth order 3 method for the performance of the system under different solution perspectives is presented. As can be noted, the system requires almost 1000 cases to reach a more stable behavior in order to ensure a suitable and constant mobility level.
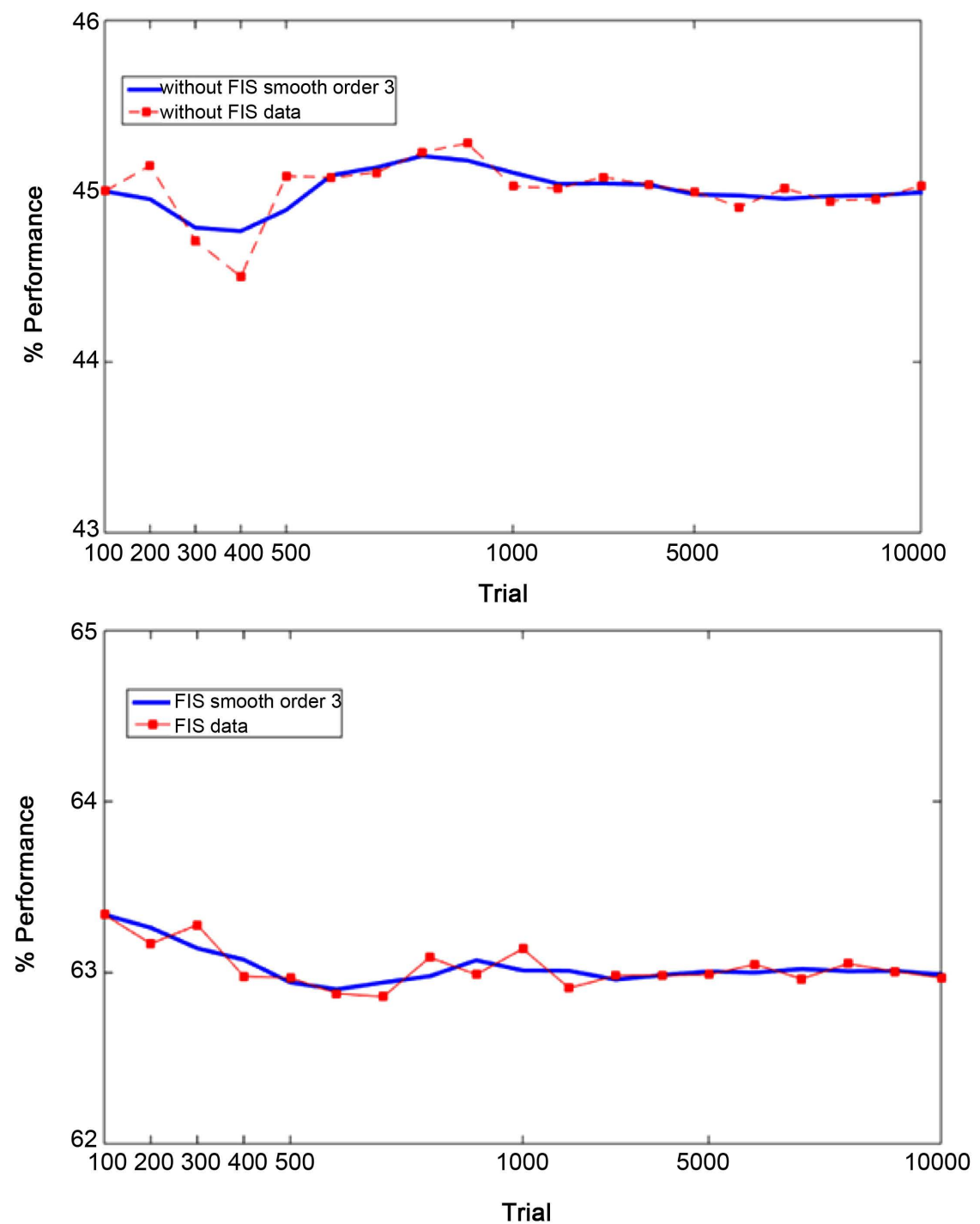

Figure 6. Performance obtained when the system uses a FIS to decide whether or not to modify the green light time. 


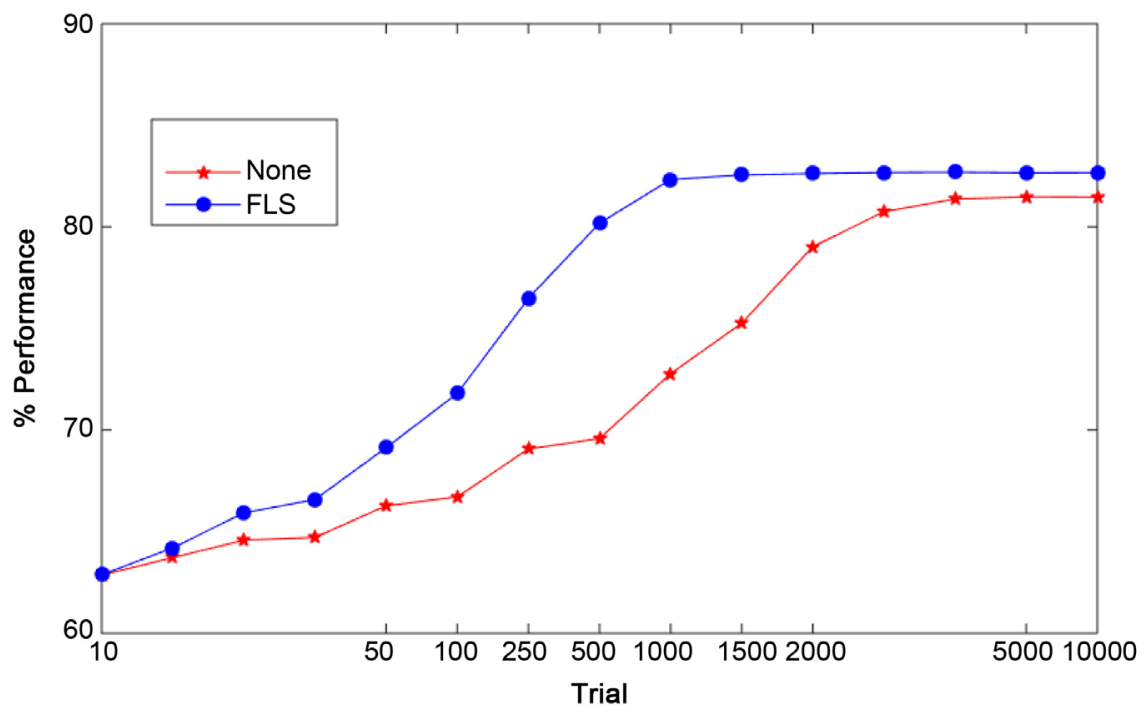

Figure 7. Comparison of the computed curve of trial experiments to reach system stability.

\section{Conclusions and Future Work}

Road traffic congestion is one of the main causes in low productivity and the decrease in modern city standards. In this sense, some recent trends in artificial intelligence suggest that in the near future, intelligent agents will improve some road challenges. In this light, the present paper introduces the use of a fuzzy inference system to define when it will be opportune to change the length of the green light in a determine traffic light signal looking for improving the level of service offered in a particular junction.

The results show that using a decision support technique, such as a fuzzy logic rules, the system performance increases in almost $18 \%$. Therefore, it is possible to identify that the use of this technique allows the system to modify the duration of the green light, improving its decision-making process which is reflected in an improvement of its operative capacity, its reliability and certainty. It is important to remark that for experimental tests, the human factor had a vital role because an expert in traffic control recommends the variables and the rules consider in the FIS. For future approaches, it is necessary considered to expand the study about which data should be taken into account in order to reach a suitable and trustworthy set of real variables.

From the obtained results, it is possible to argue that the proposed approach achieves significant benefits in vehicular mobility, in terms of congestions by increasing the traffic capacity of any intersections. However, future investigation is suggested including the following aspects: "Issues such as acceleration and des-acceleration, collision avoidance and vehicles with different velocities, must be included in the simulator by offering a set of more realistic situations in the virtual instrument proposed here".

Indeed, people are different and react modifying their behavior pattern, considering variables that have not impact on the situation. Drivers are the most 
important source of data. At the moment, the literature has not reported a system robust enough to satisfy the road constraints of the cities. Intelligent transport systems must be closely designed according to the human beings. In this light, it is necessary to develop inference models based on humans where emotions, beliefs, politics, manners, etc., can be considered. In this light, it is necessary to develop inference models based on human must consider emotions, beliefs, politics, manners, etc.

\section{References}

[1] Ibarra, S., Castán, J.A. and Laria, J. (2014) Optimizaing Urban Traffic Control Using a Rational Agent. Journal of Zhejiang University Science C, 15, 1123-1137. https://doi.org/10.1631/jzus.C1400037

[2] Zadeh, L.A. (1965) Fuzzy Sets. Information and Control, 8, 338-353. https://doi.org/10.1016/S0019-9958(65)90241-X

[3] Debnath, J., Biswas, A., Sivan, P., Sen, K.N. and Sahu, S. (2016) Fuzzy Inference Model for Assessing Occupational Risks in Construction Sites. International Journal of Industrial Ergonomics, 55, 114-128. https://doi.org/10.1016/j.ergon.2016.08.004

[4] Buemi, A., Giacalone, D., Niccari, F. and Spampinato, G. (2016) Efficient Fire Detection Using Fuzzy Logic. 6th International Conference on Consumer Electronics, Berlin, 5-7 September 2016, 47.

[5] Saner, T., Gardashova, L., Allahverdiyev, R. and Eyupoglu, S. (2016) Analysis of the Job Satisfaction Index Problem by Using Fuzzy Inference. Procedia Computer Science, 102, 45-50. https://doi.org/10.1016/j.procs.2016.09.368

[6] Skoruoski, J. and Uchronski (2016) Managing the Process of Passenger Security Control at an Airport Using the Fuzzy Inference System. Expert Systems with Applications, 54, 284-293. https://doi.org/10.1016/j.eswa.2015.11.014 